\title{
An ovarian cancer model with positive ER: Reversion of ER antagonist resistance by Src blockade
}

\author{
LONG LI $^{1 *}$, XIAOJUN LI ${ }^{2 *}$, XIAOBING HAN ${ }^{1}$, TING YANG ${ }^{1}$, JING FU $^{1}$, YUNFENG ZHANG $^{2}$ and WENLI GOU ${ }^{1}$ \\ Departments of ${ }^{1}$ Gynaecology and Obstetrics, and ${ }^{2}$ Second Thoracosurgery, The First Affiliated Hospital \\ of Xi'an Jiaotong University, Xi'an, Shaanxi 710061, P.R. China
}

Received February 14, 2014; Accepted April 16, 2014

DOI: $10.3892 /$ or.2014.3284

\begin{abstract}
Approximately $60 \%$ of ovarian cancers are positive for the estrogen receptor (ER); however, ER-targeted treatment is disappointing due to drug resistance as compared with breast cancer. In estrogen-sensitive cancers, estrogen activates Src to phosphorylate p27 promoting its degradation and increasing cell cycle progression. Since Src is frequently activated in ovarian cancers, we investigated whether combined Src and ER blockade by saracatinib and fulvestrant would circumvent anti-estrogen resistance. In 20 out of 40 enrolled patients with immunohistochemically ER-positive ovarian cancer, phosphorylated Src (p-Src) at the site of 416 tyrosine was expressed with a propensity for metastasis and a poorer disease-free survival (DFS) at 3 years following ER antagonist treatment. The effects of ER and Src blockade on cell cycle were assayed in estrogen receptor $\alpha$ $(\mathrm{ER} \alpha)$-positive ovarian cancer. We observed that Src activity was fairly greater in anti-estrogen-resistant ovarian cancer cells than that in the anti-estrogen-sensitive cell line. Estrogen activated Src via ER-Src binding and ER translocation from cytoplasm to nucleus. Mitogenesis was mediated via ER $\alpha$, not ER $\beta$. Combined saracatinib and fulvestrant increased p27 and inhibited cell cycle progression. Furthermore, dual therapy induced autophagy and inhibited ovarian cancer xenograft growth more effectively than monotherapy. Saracatinib facilitated the therapeutic effects of fulvestrant by antagonizing the estrogen-mediated Src activation. These are supportive of further preclinical assessment of combined fulvestrant and saracatinib in patients with ovarian cancer.
\end{abstract}

Correspondence to: Professor Wenli Gou, Department of Gynaecology and Obstetrics, The First Affiliated Hospital of Xi'an Jiaotong University, No. 227 Yanta West Road, Xi'an, Shaanxi 710061, P.R. China

E-mail: blue.jackie@stu.xjtu.edu.cn

${ }^{*}$ Contributed equally

Key words: ovarian cancer, saracatinib, fulvestrant, resistance

\section{Introduction}

Eighty percent of patients with ovarian cancer are diagnosed with pathological stage III/IV, suffering incurable progression within 3 years $(1,2)$. Anti-ER treatments are well-tolerated and appealing in this setting, but are frequently met with anti-estrogen resistance (3). Estrogen (E2) is implicated in the ovarian carcinogenesis. E2 drives tumor proliferation via estrogen receptor $\alpha(\mathrm{ER} \alpha)$ and anti-estrogen can markedly inhibit ovarian cancer mitogenesis (3-5). As compared with a relatively lower level of ER $\beta$ in ovarian cancers, ER $\alpha$ in ovarian cancers is expressed at a level similar to that in breast cancers, 60-70\% (6,7). Despite a relatively higher level of ER expression in ovarian cancers, numerous small clinical trials of ER-blockers were disappointing (3-6). Tamoxifen yielded a low response rate for recurrent ovarian cancers (mean, $13 \%$ ). In parallel, the pure ER-antagonist, fulvestrant, had the overall response of $\sim 10 \%$ in advanced and heavily pretreated recurrent ovarian cancer (3-5). In sum, therapeutic regimens that block pro-tumorigenic estrogen effects in ovarian cancer have not been studied in well-designed trials.

Moreover, $12-34 \%$ of ovarian cancer that do initially respond invariably and frequently develop anti-estrogen resistance $(8,9)$. Estrogen efficiently promotes transition from quiescence to G1 in ER-sensitive cells (10). Cyclin-bound cyclin-dependent kinase (Cdk) and Cdk inhibitors mutually orchester cell cyclin progression. In breast cancer cells, ER blockade is able to induce G1 phase arrest by upregulating p27 expression $(11,12)$. Notably, p27 is frequently downregulated in ovarian cancers $(13,14)$. This may result from constitutive activation of Src, which facilitates degradation of p27 by Src-induced phosphorylation. Crosstalk between estrogen-bound ER and Src drives mitogenic pathways that are frequently activated in ovarian cancer (15). On the other hand, the lack of effective treatments for recurrent ovarian cancer has stimulated development of targeted ovarian cancer therapies. Mitogenic pathways, including Src, Ras/Raf/MEK and PI3K/ AKT, are frequently activated in ovarian cancer (16). Src is overexpressed and activated in the majority of ovarian cancers and regulates cell anti- and pro-apoptosis $(17,18)$. It has been demonstrated that the intercross action between liganded ER and Src contributes to mitogenesis and ER-activated gene expression $(3,17,19)$. A potent inhibitor of $\mathrm{Abl}$ and Src family 
kinases, saracatinib (AZD0530), is able to inhibit cell invasion in vitro and xenograft growth in vivo, and is well-tolerated in phase I trial (3). Nevertheless, the anti-estrogen effect has not been evaluated in ovarian cancer.

In the present study, we investigated whether constitutive Src activation contributed to resistance to ER-blockade in ovarian cancer. We found that saracatinib reversed fulvestrant resistance in vitro and in vivo, cell cycle arrest, autophagy and apoptosis. The data provide novel insights for crosstalk between ER and Src pathways in ovarian cancer.

\section{Materials and methods}

Patients and tissue samples. A total of 40 ovarian cancers with immunohistochemically positive ER expression were selected and collected following radical surgery at the Department of Pathology of the First Affiliated Hospital of Xi'an Jiaotong University from 1999 to 2000 . The cases of ovarian cancer displayed ER-positive expression determined by two individual pathologists according to immunohistochemistry (IHC) assay. All patients in this study were classified as International Federation of Gynecology and Obstetrics (FIGO) stage III (FIGO). Subsequently, postoperative therapeutic strategy was determined by a multi-disciplinary team (MDT), including an oncosurgeon, an oncologist and a radiologist. Accordingly, ER antagonist, fulvestrant, was recommended to treat ER-positive ovarian cancer as a single agent, $500 \mathrm{mg}$ intramuscularly on day 1, $250 \mathrm{mg}$ intramuscularly on day 15 , and $250 \mathrm{mg}$ intramuscularly on day 29 and every 28 days thereafter until either in-tolerance or disease progression.

The patients with ovarian cancer were routinely scheduled for life-long follow-up. The enrolled patients were followed up at the outpatient clinic at intervals of three months during the first two years and every six months for three more years. Metastatic events were diagnosed at the outpatient clinic. Whenever a distant metastasis was suspected, radiologic, endoscopic or histologic confirmation was compulsory. The calculation of disease-free survival (DFS) started at the date of surgery and ended at the date of one of the following events: recurrence, distant metastasis or oncological death. As reported thus far, no participants were lost during follow-up.

Immunohistochemistry. After routine deparaffinization and hydration, tissue sections were treated with $3 \%$ hydrogen peroxide and heated in EDTA $(\mathrm{pH}$ 8.0) for antigen retrieval. Following serum deprivation, ER $\alpha$ and $\mathrm{p}-\mathrm{Src}$ (phospho-Y416-Src) antigen-antibody reactions took place at $4^{\circ} \mathrm{C}$ overnight. The streptavidin/peroxidase kit (Invitrogen) was used to detect antigen-antibody reactions. The purified rabbit/rat monoclonal antibodies against human ER $\alpha$ (ab-81086) and Src (ab-106271) (both from Abcam) were used at $2 \mu \mathrm{g} / \mathrm{ml}$ and goat anti-rabbit/rat biotin-conjugated IgG was secondary antibody. Immunohistochemical signals were scored by two independent observers. The scores were calculated as the number of stained cells divided by the total number of cancer cells counted. Four high-power fields $(\mathrm{x} 400)$ per slide were calculated and outcomes were averaged. Unequivocal staining of cytoplasm in $>50 \%$ of cancer cells was considered as positive.
Cell culture and transfection. Ovarian cancer cell lines, SKOV-3 and an inherent anti-estrogen-resistant variant, SKOV-3R, established after prolonged passage, were cultured in DMEM supplemented with 10\% FBS. Cells (293T) were a gift from Professor Chen Huang from the Medical College of Xi'an Jiaotong University. Cell lines in the present study were authenticated using American Type Culture Collection (ATCC) guidelines. Asynchronous culture was treated with $10^{-6} \mathrm{~mol} / \mathrm{l}$ saracatinib, $10^{-6} \mathrm{~mol} / \mathrm{l}$ fulvestrant or both for $48 \mathrm{~h}$. SKOV-3R with $0.1 \%$ cFBS for $48 \mathrm{~h}$ were then treated with ER $\alpha$ and ER $\beta$ agonists/antagonists. Then, SKOV-3R cells were transduced with lentivirus carrying pGIPZ-luc-flag encoding an Src-specific shRNA (5'-CAGATTGTCAACAACACAG-3') or control shRNA with the selection of puromycin $(8 \mu \mathrm{g} / \mathrm{ml})$ for stably transfected cells. Lentivirus-shRNA was purchased from GenePharma, Shanghai, China.

Agents and drugs. Saracatinib $\left(10^{-6} \mathrm{~mol} / \mathrm{l}\right)$ and fulvestrant $\left(10^{-6} \mathrm{~mol} / \mathrm{l}\right)$ were dissolved in dimethyl sulfoxide (DMSO) (AstraZeneca). The pure ER $\alpha$ agonist 4,40,400-[4-propyl$(1 \mathrm{H})$-pyrazole-1,3,5-triyl]trisphenol (PPT), the selective ER $\beta$ agonist 2,3-bis(4-hydroxyphenyl)-propionitrile (DPN), the dual ER $\alpha$ agonist/ER $\beta$ antagonist (R,R)-5,11-diethyl5,6,11,12-tetrahydro-2,8-chrysenediol (THC), and the ER $\beta$ antagonist 4-[2-phenyl-5,7-bis(trifluoromethyl)pyrazolo[1,5-a] pyrimidin-3-yl]-phenol (PHTPP) were obtained from GenePharma.

Immunofluorescence microscopy. Cells were serum/E2-deprived for $24 \mathrm{~h}$, then treated with $10^{-8} \mathrm{~mol} / \mathrm{l} \mathrm{E} 2$ for the indicated times. Immunofluorescence (IF) was processed via anti-ER $\alpha$ (ab-12223) or anti-p-Src (ab-4816) (both from Abcam). Cells were counterstained by 40,6-diamidino-2-phenylindole (DAPI) and analyzed by IF microscopy. Dual ER $\alpha$ and p-Src staining were linked to Texas Red for p-Src and GFP for ER $\alpha$.

Subcellular fractionation. Cellular subcellular fractionations were serum/E2-deprived for $24 \mathrm{~h}$ and then treated with E2 $\left(10^{-8} \mathrm{~mol} / \mathrm{l}\right)$ for $4 \mathrm{~h}$. Fractionation was performed as previously described (20).

Cell cycle analysis. Cells were bromodeoxyuridine (BrdUrd)labeled, counterstained with propidium iodide, and cell cycle distribution was assayed as previously described (21).

Annexin V staining. Drug-treated cells were analyzed by FITC Annexin V apoptosis detection kit I (BD Biosciences) as per the manufacturer's instructions.

Detection of autophagic vesicles. Drug effects on autophagic vesicles were evaluated by Cyto-ID Autophagy Detection kit (Enzo) as per the manufacturer's instructions.

Immunoblotting, immunoprecipitation and kinase assay. Western blot analysis was conducted and quantitated by densitometry. ER $\alpha$, MAPK, p-MAPK antibodies were purchased from Santa Cruz; p27, Akt, p-Akt, Src and p-Src antibodies from Abcam. ER-Src complexes were precipitated after $24 \mathrm{~h}$ of serum/E2 deprivation. At intervals after E2 repletion, Src and $E R \alpha$ were detected by western blot analysis. 
Xenografts. Luciferase-positive SKOV-3R cells re-suspended with Matrigel were implanted at the axillary top of female BALB/C nude mice. Xenograft growth was compared in nude mice (10 per group) with supplemental estradiol at $0.72 \mathrm{mg} / 90$ days (Invitrogen). E2-supplemented animals (10 per group) either received no treatment, fulvestrant $3.5 \mathrm{mg}$ per week SQ, fulvestrant $5 \mathrm{mg}$ per week SQ plus saracatinib $25 \mathrm{mg} / \mathrm{kg}$ daily (via oral gavage) or both starting either 11 or 26 days after tumor implantation. Viable tumor burden (luciferase activity) was assessed weekly. Mice were imaged by IVIS-100 bioluminescence imager (IVIS). Animals were weighed twice per week. Entire experimental data was detailed and documented.

Statistical analysis. All assays of cell cycle distribution, IF and $\mathrm{IP} /$ western blot analysis were at least triplicated. Xenograft studies were repeated twice. Data were characterized as means \pm SEM. One- or two-way ANOVA was conducted to assess differences between treatment groups. The significance was a statistical indication of synergism, suggesting that the combined effect of two agents was manifested in a non-additive manner. Following a significant ANOVA result $(\mathrm{P}<0.05)$ rejecting the null hypothesis, Tukey honestly significant difference (HSD) test was used for all pairwise mean comparisons. These multiple comparison procedures ensured actual error rates no greater than prespecified 5\%. Analysis was conducted in SPSS 19.0.

\section{Results}

Expression of p-Src in ER-positive ovarian cancers. Forty ER-positive ovarian cancer patients with FIGO stage III were eligible in the present study (Fig. 1A). The participating patients with ER-positive ovarian cancer received post-operative treatment of ER antagonist, fulvestrant. We observed that activated Src, p-Src, was positively expressed in 20/40 ER-positive ovarian cancer (50\%; Fig. 1C). Notably, p-Src was not significantly associated with any clinicopathological characteristics with a favorable balance between two groups demonstrated in Table I. It was unclear whether ER antagonist was absolutely beneficial for disease progression of patients with ovarian cancer. Hence, we investigated the effects of ER antagonist on disease progression in patients with ovarian cancer. As compared with ovarian cancer patients with $\mathrm{ER}^{+} \mathrm{p}-\mathrm{Src}^{+}$, ovarian cancer patients with $\mathrm{ER}^{+} \mathrm{p}-\mathrm{Src}^{-}$presented a markedly higher metastatic incidence and a significant advantage for DFS at 3 years after ER antagonist treatment $(\mathrm{P}<0.01$; Fig. $1 \mathrm{E}$ and $\mathrm{F})$, indicating the possibility that Src activation exerted the positive effects on resistance to ER antagonist associated with the propensity for metastasis or disease progression in patients with $\mathrm{ER}^{+}$ovarian cancer.

ER $\alpha$-ER binding triggers Src activation. SKOV-3R, a variant of ascites ovarian cancer SKOV-3, displayed the resistance against estrogen during prolonged culture. Both SKOV-3R and SKOV-3 expressed ER (Fig. 2A), and SKOV-3R had clearly activated Src, MEK and AKT at higher levels than those in SKOV-3 (Fig. 2A). To explore the involvement of Src in E2-stimulated ovarian cancer proliferation, SKOV-3 was E2- and serum-deprived for $48 \mathrm{~h}$ and in turn treated with
Table I. Association of p-Src expression with clinicopathological characteristics of patients with ER-positive ovarian cancer $(n=40)$.

\begin{tabular}{lcrl}
\hline & \multicolumn{2}{c}{$\mathrm{p}$-Src expression $\mathrm{n}(\%)$} & \\
\cline { 2 - 3 } $\begin{array}{l}\text { Clinicopathological } \\
\text { characteristics }\end{array}$ & Positive & Negative & P-value \\
\hline $\begin{array}{l}\text { Age (years) } \\
\leq 60\end{array}$ & $8(40.0)$ & $8(40.0)$ & $>0.05$ \\
$>60$ & $12(60.0)$ & $12(60.0)$ & \\
Median age (years) & 67 & 66 & \\
FIGO grade & & & $>0.05$ \\
$\begin{array}{l}\text { Well } \\
\text { Moderate }\end{array}$ & $3(15.0)$ & $2(10.0)$ & \\
Poor & $7(35.0)$ & $7(35.0)$ & \\
$\begin{array}{l}\text { Lymph node } \\
\text { metastasis }\end{array}$ & $10(50.0)$ & $11(55.0)$ & \\
$\begin{array}{l}\text { Positive } \\
\text { Negative }\end{array}$ & & & $>0.05$ \\
FIGO stage & $5(25.0)$ & $5(25.0)$ & \\
$\quad$ IIIA & $15(75.0)$ & $15(75.0)$ & \\
IIIB & $4(20)$ & $5(25.0)$ & $>0.05$ \\
IIIC & $11(55.0)$ & $10(50.0)$ & \\
\hline
\end{tabular}

E2 $\left(10^{-8} \mathrm{~mol} / \mathrm{l}\right)$ for the times indicated. It was observed that estrogen activated Src in SKOV-3R (Fig. 2B). ER-estrogen binding activated $\mathrm{Src}$ in ER $\alpha$ immunoprecipitation at $30 \mathrm{~min}$ after E2 triggering (Fig. 2C), supported by the observation that ER and Src co-localized in the perinuclear zone by immunofluoresence at $15 \mathrm{~min}$ (Fig. 2D). Thus, it was likely that E2 stimulated ER-Src interaction and was subject to Src activation, which may contribute to its mitogenic effects. Furthermore, subcellular fractionation analysis showed that $\mathrm{ER} \alpha$ was strongly cytoplasmic in the E2-deprived cells, but rapidly transferred into the nucleus $48 \mathrm{~h}$ after E2 addition (Fig. 2E). As compared with breast cancer where ER was predominantly nuclear distribution $(11,12)$, the ovarian cancer cell line expressed cytoplasmic ER in the absence of E2, which translocated to the nucleus with E2 addition.

E2 enhances ovarian cancer growth via the receptor of ER . Subsequently, estrogen deprivation with or without growth factor depletion was examined. SKOV-3R growth was not inhibited after $48 \mathrm{~h}$ of E2 depletion alone (media, $10 \% \mathrm{cFBS}$ ), but estrogen and serum depleted media ( $0.1 \% \mathrm{cFBS})$ for $48 \mathrm{~h}$ diminished the percentage of S-phase cells from 50 to $15 \%$ ("P $<0.01$ vs. $10 \%$ FBS or $10 \%$ cFBS; Fig. 3A). It was of note that the addition of $\mathrm{E} 2$ alone restored $0.1 \% \mathrm{cFBS}$-induced growth arrest (Fig. 3A). It indicated that estrogen alone was able to maintain SKOV-3R cell proliferation in the absence of growth factors. To examine the ability of E2 to drive SKOV-3R cell proliferation, SKOV-3R cells were preliminarily arrested in $0.1 \%$ cFBS for $48 \mathrm{~h}$. Then, the addition of E2 alone stimulated cell cycle re-entry with \% S-phase peaking at $20 \mathrm{~h}$ (Fig. 3B). Owing to binding of either E2 or fulvestrant to ERs, including 
A

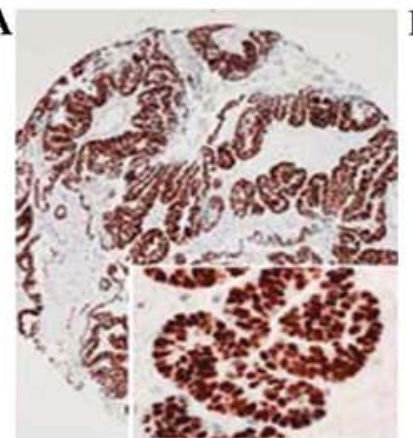

C
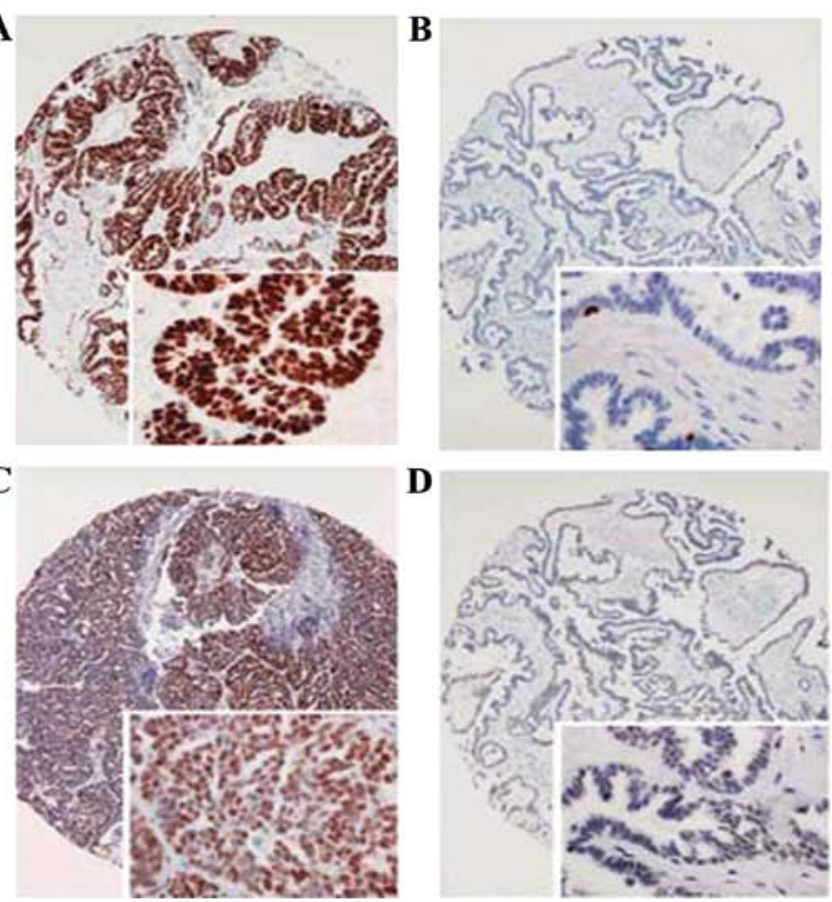

D

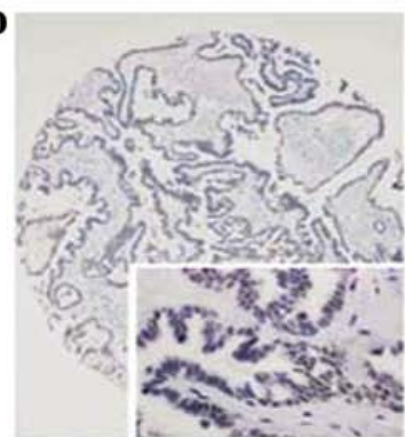

E
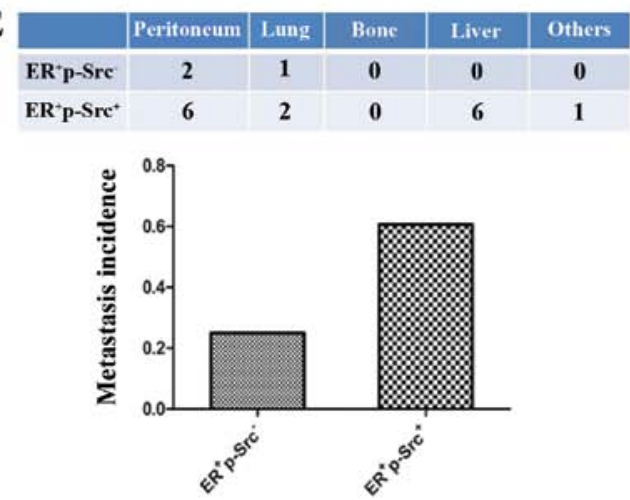

$\mathbf{F}$

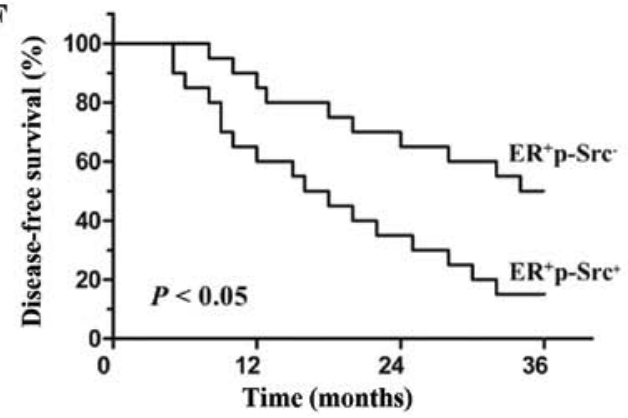

Figure 1. Expression of p-Src in ER-positive ovarian cancers. (A-D) Immunohistochemical stains for ER and p-Src were performed with low magnification (x4) and the inset with high magnification (x40). (A) ER-positive ovarian cancer; (B) ER-negative ovarian cancer; (C) p-Src-positive ovarian cancer (D) p-Src-negative ovarian cancer; (E) metastatic incidence in ER-positive ovarian cancer; (F) Kaplan-Meier survival curve for 40 patients with ER-positive ovarian cancer with or without p-Src expression. p-Src, phosphorylated Src.

$\mathrm{ER} \alpha$ and $\mathrm{ER} \beta$, we tested which receptor induced SKOV-3R cell growth arrest of estrogen. SKOV-3R cells were transferred to $0.1 \% \mathrm{cFBS}$ for $48 \mathrm{~h}$, then treated with various hormone receptor agonists or antagonists for $48 \mathrm{~h}$ before cell cycle analysis. The pure ER $\alpha$ agonist, PPT and the ER $\alpha$ agonist/ER $\beta$ antagonist, THC, rescued E2 deprivation in SKOV-3R cells. Furthermore,
A

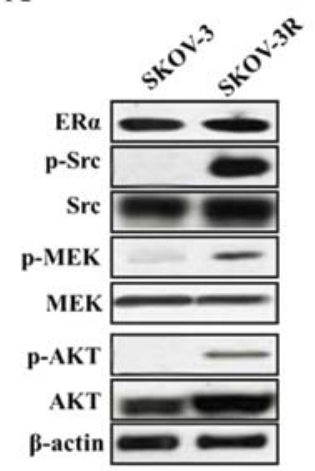

B

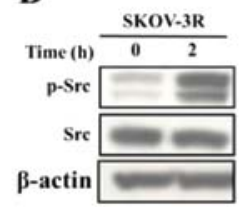

C

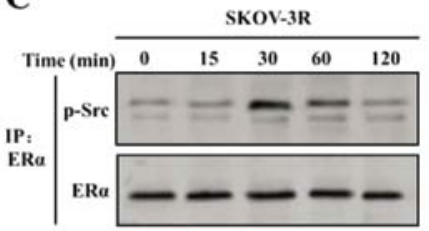

D

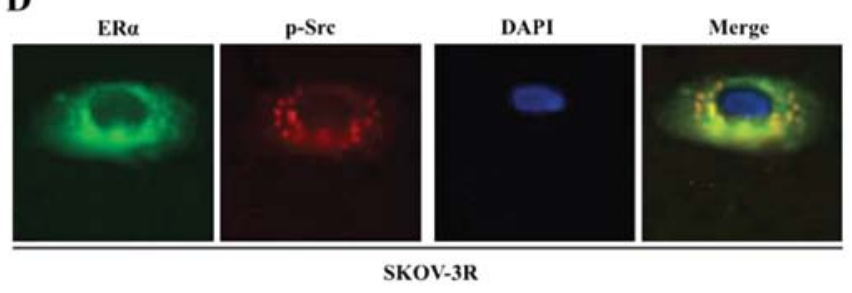

$\mathbf{E}$

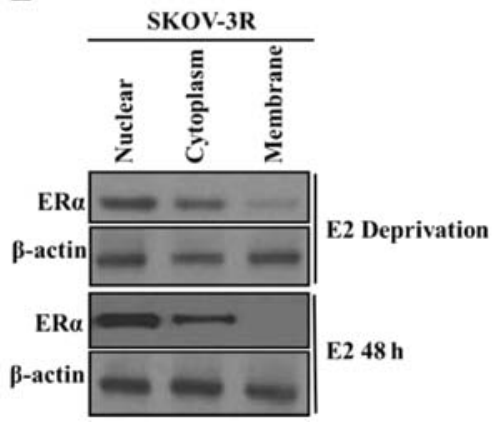

Figure 2. Estrogen-induced biological alteration: Src activation, ER/Src binding and ER translocation. (A) Western blot analysis showed ER $\alpha$, total and activated Src, MEK and AKT in SKOV-3 and SKOV-3R. (B) Western blot analysis showed phosphorylated and total $\mathrm{Src}$ at the indicated times. (C) ER $\alpha$ immunoprecipitates were immunoblotted for p-Src or ER $\alpha$. (D) Immunofluorescence (IF) showed perinuclear ER $\alpha$ and p-Src colocalization after E2 addition. (E) Subcellular fractions were blotted for ER $\alpha$ and $\beta$-actin.

the ER $\beta$ agonist, DPN, did not induce tumor cell growth while the ER $\beta$ antagonist PHTPP with E2 addition did not abrogate E2-inducing cell growth (Fig. 3A). The mean \% S-phase did not significantly differ between control and those treated with E2, PPT, THC or PHTPP, nor did they differ between DPN-treated and E2-deprived in SKOV-3R cells. Thus, the proliferative function of E2 in ovarian cancer was determined by ER $\alpha$ independent of ER $\beta$. Then, E2 function was further assayed in nude mice models in vivo (Fig. 3C). After SKOV-3R cell pellets were implanted with or without E2 manipulation, estrogen-supplemented xenographs showed considerable growth with markedly higher photon flux/sec at 16 weeks as compared with those without E2 treatment (Fig. 3C).

Saracatinib reverses resistance to fulvestrant via Src inhibition. On the basis of observation that estrogen rapidly activated Src, it was hypothesized that combined Src and ER blockade would arrest tumor growth more efficiently than 
A

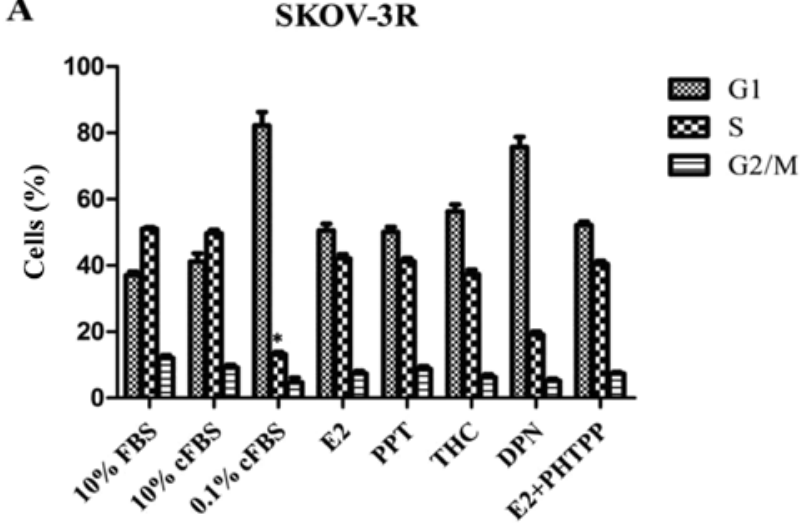

B

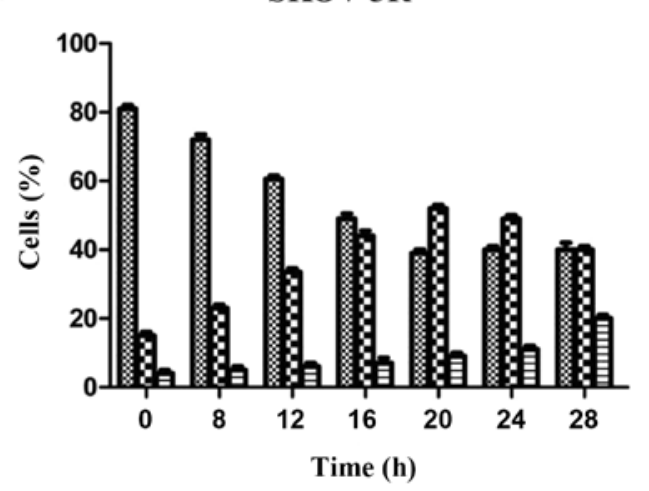

C

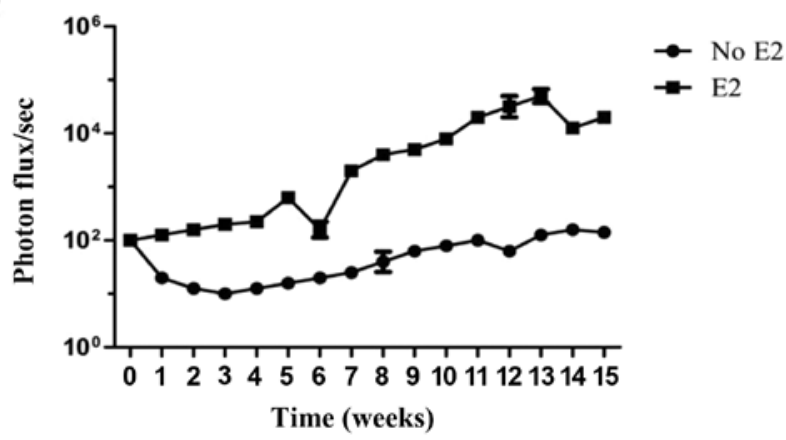

Figure 3. Estrogen-induced cell growth via ER $\alpha$. (A) SKOV-3R cells in $10 \%$ FBS were transferred to media with $10 \% \mathrm{cFBS}, 0.1 \% \mathrm{cFBS}$ alone or $0.1 \%$ cFBS together with E2, PPT, THC, DPN or E2+PHTPP for $48 \mathrm{~h}$ and cell-cycle distribution was analyzed. (B) SKOV-3R cells were E2-deprived in $0.1 \%$ cFBS for $48 \mathrm{~h}$, then E2 was supplemented for cell cycle analysis at the indicated times. (C) SKOV-3R-luct xenograft growth was assayed by IVIS. All graphs are shown as means \pm SEM.

either alone. SKOV-3R was treated with an inhibitor specific for Src, saracatinib, fulvestrant or both. There was no cell cycle arrest after $48 \mathrm{~h}$ of fulvestrant (Fig. 4A). A modest decrease of $\% \mathrm{~S}$-phase cells was detected by using saracatinib alone (Fig. 4A). Combined Src and ER blockade significantly reduced \% S-phase cells as compared with Src-specific inhibitor alone ( $\mathrm{P}<0.05$ vs. saracatinib alone group; Fig. 4A). In SKOV-3R cells, the reduction in \% S-phase cells following dual therapy was much more than a sum of decreases of either saracatinib or fulvestrant alone. It was reported that estrogen efficiently activated Src to promote p27 proteolysis via the phosphorylation of activated Src (22). Therefore, we investigated the expressions of activated Src and p27 in SKOV-3R
A

SKOV-3R
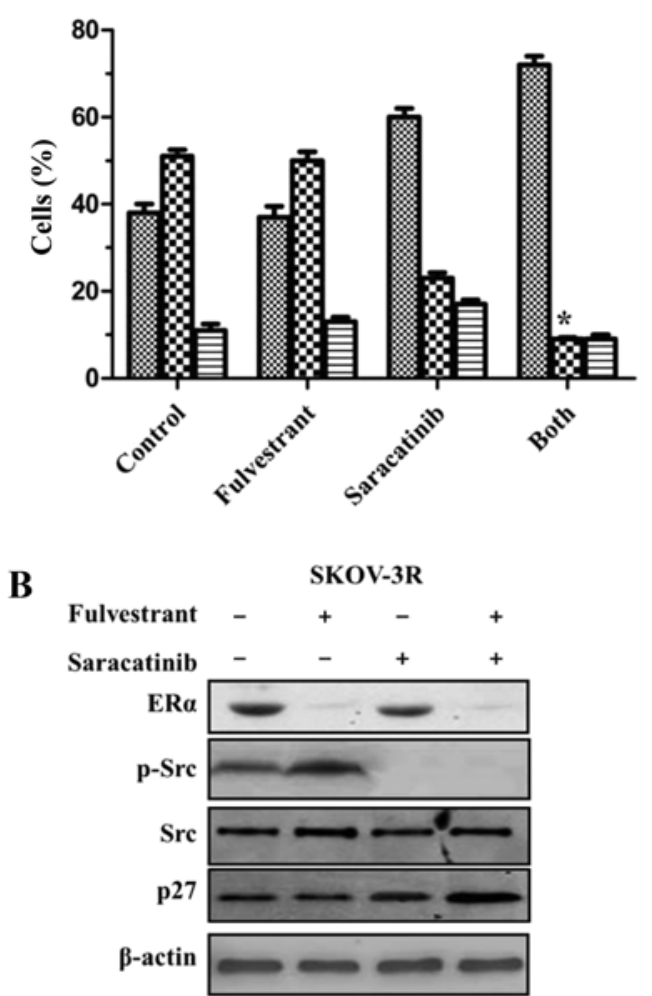

C

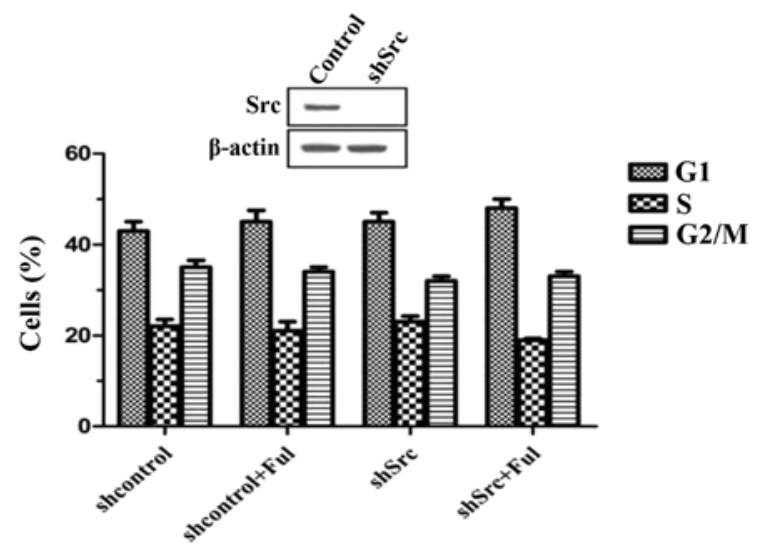

Figure 4. Combined ER and Src blockade on cell cycle. (A) SKOV-3R cell-cycle distribution after 48 h. $\mathrm{P}<0.05$ vs. Saracatinib. (B) Western blot analysis for $\mathrm{ER} \alpha$, total and activated Src and p27 in SKOV-3R cells treated by single or dual drugs. (C) SKOV-3R cells were stably transduced with shRNA-Src or shRNA-control and treated by fulvestrant after $48 \mathrm{~h}$. Western blot analysis showed Src knockdown. Cell cycle distribution is graphed as means \pm SEM.

cells. It was demonstrated that activated Src, phospho-Y416 $\mathrm{Src}(\mathrm{p}-\mathrm{Src})$, was reduced with no change in total Src in saracatinib-treated SKOV-3R (Fig. 4B). Both drugs together significantly increased p27 expression as compared with either drug alone (Fig. 4B), indicating that there was a significant synergistic interaction between fulvestrant and saracatinib for cell growth arrest via Src-regulated p27 expression.

To test the contribution of Src to saracatinib effects, SKOV-3R was stably transduced with shRNA to knockout Src $(\mathrm{shSrc})$, the contribution of fulvestrant was in turn evaluated. Although saracatinib weakened SKOV-3R cell growth (Fig. 4C), the lack of Src had little effect on cell cycle arrest as compared with control cells (Fig. 4C). Thus, it was 
$\mathbf{A}$
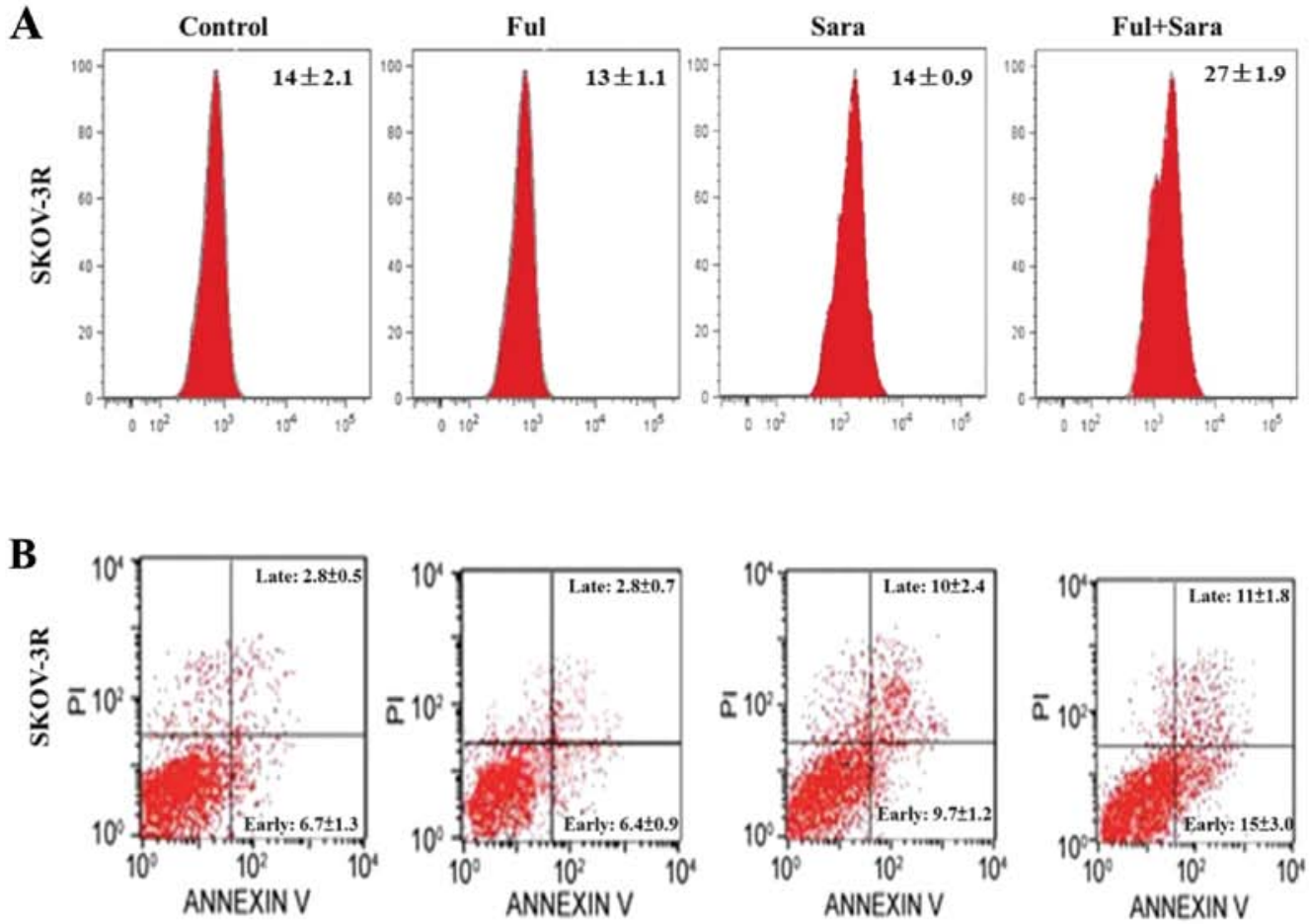

C
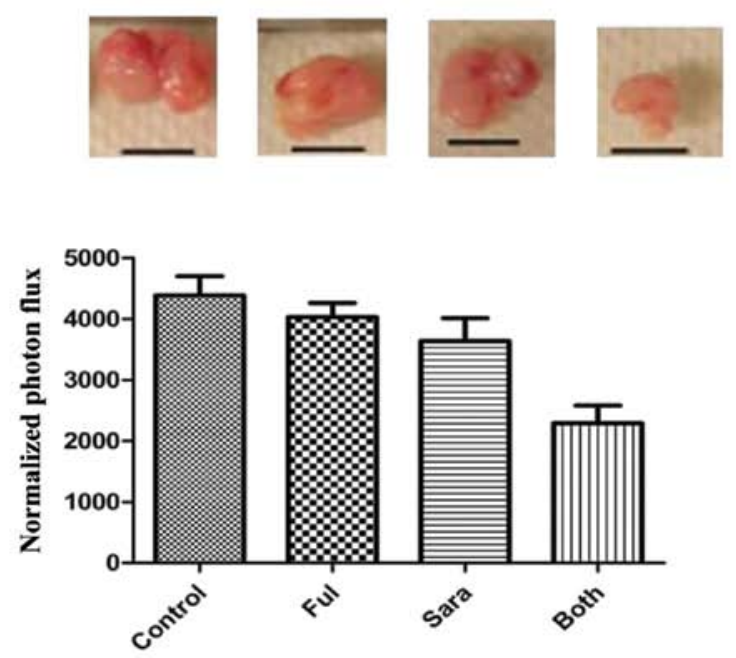

Figure 5. Dual inhibition of ER and Src affects cell apoptosis and autophagy. (A) Cyto-ID autophagy analysis. (B) SKOV-3R cells for early/late apoptosis analysis by flow cytometry. (C) Drug effects initiated with fulvestrant, saracatinib or both at day 11. Tumor growth was graphed as mean normalized photon flux (means \pm SEM).

likely that saracatinib effects were partially ascribed to the inhibition of other Src family members. Nevertheless, shSrctransfected cells with the addition of fulvestrant reduced SKOV-3R cell growth by a one-third cut of \% S-phase cells, suggesting Src knockout facilitated the fulvestrant treatment to some extent. Since Src downregulation did not produce an equal effect to saracatinib alone, it was supposed that the antiproliferative effects of saracatinib were partially due to its inhibition of other Src family members.

Src inhibition in combination with ER blockade causes cell death. Anticancer efficacy results from dual directions, including antiproliferation or cell death. We assayed autophagy and apoptosis by flow cytometry following $48 \mathrm{~h}$ treatment of saracatinib, fulvestrant or both. To examine autophagy,
SKOV-3R cells were labeled with Cyto-ID-Green autophagy dye, that was examined by flow cytometry. Accordingly, fulvestrant or saracatinib alone did not increase autophagic vesicle formation, whereas autophagic vesicles increased significantly in SKOV-3R (Fig. 5A; $\mathrm{P}<0.01$ ). In parallel, saracatinib-associated SKOV-3R growth inhibition resulted from increased events of early and late apoptosis. (Fig. 5B; P<0.05, control vs. saracatinib), and combined therapy had a significantly additional effect relative to saracatinib alone (Fig. 5B; $\mathrm{P}<0.05$, both vs. saracatinib). We showed the combined drug effects in nude mice model in vivo. Implanted tumors were harvested at 11 weeks after implantation. Regarding the combination effects, combination of fulvestrant with saracatinib significantly decreased tumor growth as compared with either drug alone (Fig. 5C). 


\section{Discussion}

More than $55 \%$ of ovarian cancers express ER on the cell surface $(23,24)$. Although ER expression in ovarian cancer is similar to breast cancer, the efforts to target ER merely present a short-term benefit in $28 \%$ of ovarian cancer cases $(4,8,9)$. In patients with breast cancer, the survival benefit of ER blockade has been confirmed in the ER-positive breast cancers $(25,26)$. Therefore, the potential efficacy of anti-estrogen in ovarian cancer may be underestimated. Numerous small trials of ER-blockers have been disappointing $(3,6)$. Only $8 \%$ overall response and $35 \%$ disease stabilization were detected in advanced heavily pretreated ovarian cancer cases (4). Hence, understanding the mechanisms of anti-estrogen resistance may provide new insight for the treatment of ovarian cancer. In estrogen-sensitive cancers, estrogen is able to stimulate Src activation to phosphorylate p27 promoting its degradation and increasing cell cycle progression (22). Crosstalk between estrogen-bound ER and Src drives mitogenic pathways that are frequently activated in ovarian cancer (15). We observed that activated Src was positively expressed in 20 out of 40 ER-positive ovarian cancer (50\%). It was unclear whether ER antagonist was beneficial for patients with $\mathrm{ER}^{+}$ovarian cancer. According to subgroup survival analysis on the basis of p-Src status in ovarian cancer, the cohort with $\mathrm{ER}^{+} \mathrm{p}-\mathrm{Src}^{-}$possessed a propensity for metastasis and an advantage for DFS at 3 years after ER antagonist treatment as compared with ovarian cancer patients with $\mathrm{ER}^{+} \mathrm{p}-\mathrm{Src}^{+}$, indicating the possibility that $\mathrm{Src}$ activation exerted the effects on underestimating therapeutic efficacy by resistance to ER antagonist in patients with $\mathrm{ER}^{+}$ ovarian cancer.

Src has potential to promote the metastatic and survival effects of cancer cells, while Src acts to promote the degradation of p27, a G1 arrestor of anti-estrogen, by phosphorylation $(11,22)$. p27 is essential for cell arrest by anti-estrogen, and Src promotes p27 proteolysis to eliminate the effect of p27 on cell arrest. Therefore, it was hypothesized that Src inhibitor, saracatinib, could restore anti-estrogen in resistant ER-positive ovarian cancers. In the present study, the data demonstrated the therapeutic potential of combined saracatinib and fulvestrant in ovarian cancer with resistance to ER antagonist. As reported by Simpkins et al (3) 338 primary ovarian cancers confirmed that $67 \%$ had detectable ER expression with p-Src (activated Src, phospho-Y416-Src) and total Src. Supporting the relevance of Src with ER, phosphorylated Src in ER-positive SKOV-3R expressed at a higher level than that in SKOV-3. It was reported that estrogen triggering ER signaling was potential to activate Src kinase in breast epithelial cells $(27,28)$. Despite the presence of ER $\alpha$ and ER $\beta$ on the surface of ovarian cancer cells, E2-stimulated SKOV-3R cell proliferation was determined by $\mathrm{ER} \alpha$ alone. In addition, it was observed for the first time that estrogen rapidly activated $\mathrm{Src}$, Src-ER interaction and their co-localization in the perinuclear cytoplasm in ovarian cancer. It was inferred that the rapid ER-Src interaction may be linked to ER-mediated transcription, that was confirmed by the observation that estrogen-stimulated Src activation was impeded by saracatinib by downregulating ER expression at two ER-modified genes: Fosl and c-Myc $(3,4)$.
Notably, cell arrest was greater for combined fulvestrant and saracatinib than either alone. Dual therapy contributed to more antiproliferative efficacy compared to monotherapy. Furthermore, a more significant growth arrest was caused by combined blockade of Src and ER with a greater increase in p27 than that with monotherapy in SKOV-3R cells. In SKOV3R, fulvestrant and saracatinib may synergistically block cell cycle by upregulating p27 and activating p27-related signaling. The outcomes were consistent with recent reports that $\mathrm{Src}$ blockade intensifies anti-estrogen efficacy in breast cancers (29-31). To grow and culture tumor cells from primary ovarian cancers may allow future prediction of individualized use of targeted therapies.

Saracatinib, Src kinase inhibitor, suppressed a variety of Src family members (32). As compared with treatment of saracatinib, Src silence failed to inhibit cell growth, indicating other Src family members may be ascribed to drug efficacy. Although neither fulvestrant alone nor shSrc inhibited cell cycle, Src silence co-worked with ER blockade to efficiently hinder cell cycle progression, suggesting that Src and ER led cell growth in ER-positive ovarian cancer. Accordingly, Src and ER blockade impaired tumor cell growth, not only via antiproliferation, but also via autophagy. Autophagy is characterized as leading to type II programmed cell death (33-35). Fulvestrant alone was unable to efficiently induce autophagy in the present study, while dual drugs yielded the greatest effect on inducing autophagy relative to single drugs. Thus, autophagic cell death was promoted by Src inhibition and augmented by the extra addition of ER blockade. Src inhibitorinduced autophagy accounted for saracatinib-mediated antitumor effect.

We have characterized a new model for the study of antiestrogen resistance in ovarian cancer. SKOV-3R was markedly anti-estrogen resistant, but retained estrogen sensitivity in vitro and in vivo. SKOV-3R xenografts required estrogen for growth. Implantation of luciferase-tagged SKOV-3R allowed highly sensitive and quantitative monitoring of ovarian tumor growth. While short-term saracatinib was effective in vitro, resistance rapidly emerged in vivo. Estrogen-driven Src activation and upregulation of other Src family kinases may all contribute to failure of saracatinib alone. It was noteworthy that, when combined with fulvestrant, saracatinib resistance did not occur in ovarian cancer SKOV-3R xenografts. While most targeted therapies reduced tumor growth, combined saracatinib and fulvestrant caused tumor regression. The late regression reflected the induction of autophagy leading to loss of tumor viability. It was highly likely that the greater antitumor effect of dual therapy resulted from more effective inhibition of estrogen-activated gene expression in order to facilitate a considerable number of cell deaths.

Ovarian cancer is greatly unresponsive to hormonal therapies. However, our results raised the possibility that ER and Src blockade prevented or reversed anti-estrogen resistance in ovarian cancer. Well-designed pre-clinical evaluation of dual drug combination is deemed as necessary.

\section{Acknowledgements}

This study was supported by the Key Science and Technology Program of Shaanxi Province (no. 2011K13-01-13). 


\section{References}

1. Siegel R, Naishadham D and Jemal A: Cancer statistics, 2013 CA Cancer J Clin 63: 11-30, 2013.

2. Siegel R, Naishadham D and Jemal A: Cancer statistics, 2012. CA Cancer J Clin 62: 10-29, 2012.

3. Simpkins F, Hevia-Paez P, Sun J, et al: Src inhibition with saracatinib reverses fulvestrant resistance in ER-positive ovarian cancer models in vitro and in vivo. Clin Cancer Res 18: 5911-5923, 2012.

4. Argenta PA, Um I, Kay C, et al: Predicting response to the anti-estrogen fulvestrant in recurrent ovarian cancer. Gynecol Oncol 131: 368-373, 2013.

5. Badia E, Docquier A, Busson M, et al: Long-term treatment with the pure anti-estrogen fulvestrant durably remodels estrogen signaling in BG-1 ovarian cancer cells. J Steroid Biochem Mol Biol 132: 176-185, 2012.

6. Bossard C, Busson M, Vindrieux D, et al: Potential role of estrogen receptor beta as a tumor suppressor of epithelial ovarian cancer. PLoS One 7: e44787, 2012.

7. Halon A, Materna V, Drag-Zalesinska M, et al: Estrogen receptor alpha expression in ovarian cancer predicts longer overall survival. Pathol Oncol Res 17: 511-518, 2011.

8. Kothari R, Argenta P, Fowler J, Carter J and Shimp W: Antiestrogen therapy in recurrent ovarian cancer resulting in 28 months of stable disease: a case report and review of the literature. Arch Oncol 18: 32-35, 2010.

9. Smyth JF, Gourley C, Walker G, et al: Antiestrogen therapy is active in selected ovarian cancer cases: the use of letrozole in estrogen receptor-positive patients. Clin Cancer Res 13: 3617-3622, 2007.

10. Park MA, Hwang KA, Lee HR, Yi BR, Jeung EB and Choi KC: Benzophenone-1 stimulated the growth of BG-1 ovarian cancer cells by cell cycle regulation via an estrogen receptor alpha-mediated signaling pathway in cellular and xenograft mouse models. Toxicology 305: 41-48, 2013.

11. Wang L, Wang G, Yang D, et al: Euphol arrests breast cancer cells at the G1 phase through the modulation of cyclin D1, p21 and p27 expression. Mol Med Rep 8: 1279-1285, 2013.

12. Jiang D, Wang X, Liu X and Li F: Gene delivery of cyclin-dependent kinase inhibitors $p 21^{\text {Wafl }}$ and $p 27^{\text {Kipl }}$ suppresses proliferation of MCF-7 breast cancer cells in vitro. Breast Cancer 2013 [Epub ahead of print].

13. Psyrri A, Bamias A, Yu Z, et al: Subcellular localization and protein levels of cyclin-dependent kinase inhibitor p27 independently predict for survival in epithelial ovarian cancer. Clin Cancer Res 11: 8384-8390, 2005.

14. Hurteau JA, Allison BM, Brutkiewicz SA, et al: Expression and subcellular localization of the cyclin-dependent kinase inhibitor p2 $7^{\mathrm{Kipl}}$ in epithelial ovarian cancer. Gynecol Oncol 83: 292-298, 2001.

15. Xing D and Orsulic S: A genetically defined mouse ovarian carcinoma model for the molecular characterization of pathwaytargeted therapy and tumor resistance. Proc Natl Acad Sci USA 102: 6936-6941, 2005.

16. Integrated genomic analyses of ovarian carcinoma. Nature 474: 609-615, 2011.

17. Huang YW, Chen C, Xu MM, Li JD, Xiao J and Zhu XF: Expression of c-Src and phospho-Src in epithelial ovarian carcinoma. Mol Cell Biochem 376: 73-79, 2013.
18. Kim HS, Han HD, Armaiz-Pena GN, et al: Functional roles of Src and Fgr in ovarian carcinoma. Clin Cancer Res 17: 1713-1721, 2011.

19. Matsuo K, Nishimura M, Bottsford-Miller JN, et al: Targeting SRC in mucinous ovarian carcinoma. Clin Cancer Res 17: 5367-5378, 2011.

20. Zhao Y, Li X, Sun X, Zhang Y and Ren H: EMT phenotype is induced by increased Src kinase activity via Src-mediated caspase-8 phosphorylation. Cell Physiol Biochem 29: 341-352, 2012.

21. Castoria G, Giovannelli P, Lombardi M, et al: Tyrosine phosphorylation of estradiol receptor by Src regulates its hormone-dependent nuclear export and cell cycle progression in breast cancer cells. Oncogene 31: 4868-4877, 2012.

22. Chu I, Sun J, Arnaout A, et al: p27 phosphorylation by Src regulates inhibition of cyclin E-Cdk2. Cell 128: 281-294, 2007.

23. Serkies K, Sinacki M and Jassem J: The role of hormonal factors and endocrine therapy in ovarian cancer. Contemp Oncol 17: 14-19, 2013

24. Brenton JD and Stingl J: Stem cells: anatomy of an ovarian cancer. Nature 495: 183-184, 2013.

25. Coates AS, Keshaviah A, Thürlimann B, et al: Five years of letrozole compared with tamoxifen as initial adjuvant therapy for postmenopausal women with endocrine-responsive early breast cancer: update of study BIG 1-98. J Clin Oncol 25: 486-492, 2007.

26. Regan MM, Neven P, Giobbie-Hurder A, et al: Assessment of letrozole and tamoxifen alone and in sequence for postmenopausal women with steroid hormone receptor-positive breast cancer: the BIG 1-98 randomised clinical trial at 8.1 years median follow-up. Lancet Oncol 12: 1101-1108, 2011.

27. Jung J, Kim HY, Kim M, Sohn K, Kim M and Lee K: Translationally controlled tumor protein induces human breast epithelial cell transformation through the activation of Src. Oncogene 30: 2264-2274, 2011.

28. Hui AY, Meens JA, Schick C, et al: Src and FAK mediate cell-matrix adhesion-dependent activation of Met during transformation of breast epithelial cells. J Cell Biochem 107: 1168-1181, 2009.

29. Nautiyal J, Yu Y, Aboukameel A, et al: ErbB-inhibitory protein: a modified ectodomain of epidermal growth factor receptor synergizes with dasatinib to inhibit growth of breast cancer cells. Mol Cancer Ther 9: 1503-1514, 2010.

30. Moulder S, Yan K, Huang F, et al: Development of candidate genomic markers to select breast cancer patients for dasatinib therapy. Mol Cancer Ther 9: 1120-1127, 2010.

31. Gucalp A, Sparano JA, Caravelli J, et al: Phase II trial of saracatinib (AZD0530), an oral SRC-inhibitor for the treatment of patients with hormone receptor-negative metastatic breast cancer. Clin Breast Cancer 11: 306-311, 2011.

32. Arcaroli JJ, Touban BM, Tan AC, et al: Gene array and fluorescence in situ hybridization biomarkers of activity of saracatinib (AZD0530), a Src inhibitor, in a preclinical model of colorectal cancer. Clin Cancer Res 16: 4165-4177, 2010.

33. Boya P, Reggiori $\mathrm{F}$ and Codogno P: Emerging regulation and functions of autophagy. Nat Cell Biol 15: 713-720, 2013.

34. Choi AM, Ryter SW and Levine B: Autophagy in human health and disease. N Engl J Med 368: 651-662, 2013.

35. Fimia GM, Corazzari M, Antonioli M and Piacentini M: Ambra1 at the crossroad between autophagy and cell death. Oncogene 32: 3311-3318, 2013. 